\title{
ANÁLISE DE CONSULTAS SQL E CYPHER EM DADOS DE PRODUÇÃO AGRÍCOLA
}

\author{
Rebeca Einhardt Fiss ${ }^{1}$, Ana Paula Lüdtke Ferreira ${ }^{2}$ e Naylor Bastiani Perez ${ }^{3}$ \\ ${ }^{I}$ IF sul Campus Santana do Livramento, PPGCAP Unipampa, Santana do Livramento, RS, Brasil \\ ${ }^{2} P P G C A P$ Unipampa, Bagé, RS, Brasil \\ ${ }^{3}$ Embrapa Pecuária Sul, PPGCAP Unipampa, Bagé, RS, Brasil
}

\begin{abstract}
RESUMO
Os dados provenientes dos sistemas de produção agropecuária diferem dos dados usados em organizações mais tradicionais: variações espaciais e temporais referentes aos atributos das entidades do sistema devem ser mantidas, novos atributos podem ser inseridos a qualquer tempo, nem todos os valores de atributos são coletados nos mesmos instantes e a busca pode ser feita por valores e não necessariamente pelas chaves de acesso. A pouca estrutura e a busca por valores armazenados implicam em um maior custo computacional nas operações de gerenciamento do banco e consultas, se a implementação é feita no âmbito dos sistemas gerenciadores de bancos de dados relacionais. Este trabalho apresenta uma análise do desempenho de bancos de dados relacionais e orientados a grafos. Os resultados mostram a superioridade esperada do modelo SQL na inserção de dados, enquanto que o modelo orientado a grafos realiza consultas mais eficientes quando os elementos procurados não são chaves de busca.
\end{abstract}

\section{PALAVRAS-CHAVE}

Agricultura de Precisão, Complexidade computacional, Neo4j, MySQL

\section{INTRODUÇÃO}

A agricultura de precisão exige a coleta de dados do sistema produtivo para subsidiar as decisões de gerenciamento relacionadas à variabilidade espacial e temporal dos resultados de produção (MAPA, 2011). Os dados provenientes de sistemas de produção agropecuária, contudo, diferem daqueles de empreendimentos industriais, comerciais, educacionais e outros que fazem uso de grandes quantidades de dados. As informações sobre esses domínios são estruturadas, as entidades têm um conjunto fixo e limitado de atributos e relacionamentos e as chaves de busca são facilmente estabelecidas. O agronegócio faz uso de informações organizadas, mas com diferenças importantes que podem ser enunciadas como: (i) os dados coletados têm características espaciais e/ou temporais, exigindo o georreferenciamento e timestamps de coleta, (ii) o conjunto de atributos de uma entidade não é fixo e nem limitado, (iii) chaves de acesso são difíceis de definir, pois as informações precisam ser recuperadas em termos de valores de atributos; (iv) valores são coletados em diferentes pontos de tempo e espaço, nem sempre juntas ou com a mesma frequência; (v) novos atributos podem ser criados a qualquer momento, a partir da disponibilidade de novos dados. Os modelos relacionais podem ser adaptados para lidar com esses requisitos, mas essa adaptação resulta em uma coleção dispersa de tabelas com poucos atributos, usualmente em uma estrutura chave-valor. A combinação de tabelas para encontrar resultados - como, por exemplo, características de todas as áreas plantadas com produção menor que certo valor - gera consultas SQL ineficientes, visto que as operações de junção são executadas usando o produto cartesiano de tuplas com atributos selecionados (Date, 2003).

Durante os anos 1990, bases de dados fora do padrão de esquemas e consultas SQL começaram a ser propostas, com o objetivo de flexibilizar os esquemas rígidos de descrição de dados do modelo relacional (Robinson et. al., 2015). Essas bases, conhecidas como NOSQL (Not Only SQL), permitem que qualquer novo tipo de dado possa ser associado com um registro já existente. A literatura apresenta análises de desempenho dos bancos de dados NoSQL, em relação aos bancos de dados relacionais ou entre si: PostgreSQL e MongoDB para dados espaciais (Laksono, 2018) ou climáticos (Lian et. al., 2018); relacionais 
e de grafos, usando os bancos JanusGraph e TigerGraph para dados espaço-temporais (Baker Effendi et. al., 2020). Todos os trabalhos concluem pela superioridade dos bancos não relacionais.

A hipótese de pesquisa presente neste trabalho é que bases de dados orientadas a grafos são mais apropriadas para armazenamento e consulta de dados agropecuários, visto que as relações estabelecidas entre os nodos do grafo são bidirecionais. O objetivo específico deste trabalho é analisar e comparar o desempenho das operações de inserção e consulta de dados de produção agropecuária com características espaço-temporais em bancos de dados relacionais e dos bancos de dados orientados a grafos. O trabalho está organizado como se segue: a Seção 2 apresenta o ferramental e os procedimentos metodológicos usados no desenvolvimento deste trabalho; a Seção 3 apresenta e discute os resultados obtidos, com fechamento do trabalho na Seção 4.

\section{MATERIAL E MÉTODOS}

O trabalho foi desenvolvido com o SGBD relacional MySQL (MySQL, 2020) e com o banco NOSQL Neo4j, escolhidos por sua disponibilidade, uso comercial e implementações completas das propriedades ACID (Atomicity - atomicidade, Consistency consistência, Isolation - isolamento, Durability - persistência) (Data, 2013; Robinson et. al., 2015). A automatização da geração de dados e das operações de inserção e consulta aos bancos foi realizada por meio da linguagem Python (Python, 2020). As versões de banco usadas foram MySQL Community Server 8.0.19 e o Neo4J 3.5.16, em conjunto com o plugin Neo4J Spatial 0.26.2. As consultas foram realizadas, respectivamente, com as linguagens de consulta SQL e Cypher. Os gráficos para ilustração dos resultados foram desenvolvidos com a linguagem R (R Core Team, 2013). Todos as figuras apresentadas foram produzidas pelos autores. Os testes foram realizados utilizando um computador portátil, com sistema operacional Windows 10, processador Core i5 $2.5 \mathrm{GHz} 2.71 \mathrm{GHz}$ e 8 GB de memória RAM.

Como o foco é restrito à análise do tempo de execução, os valores inseridos foram gerados aleatoriamente, dentro de uma estrutura espaço-temporal - dados georreferenciados e com informações sobre o período de coleta - com as tabelas/nodos representando as variáveis agropecuárias de interesse. As Figuras 1 (a) e (b) apresentam os modelos de dados de grafos e relacional, respectivamente. No banco de dados de grafos, um nodo do tipo location armazena as coordenadas geográficas dos pontos de interesse. Ao nodo de localização é criado um arco rotulado pela data de medição a um nodo tipificado como uma variável que contém o seu valor. Dessa forma, novas variáveis e novas medições podem ser inseridas de forma independente. $\mathrm{O}$ modelo de dados relacional contém uma tabela para cada variável de interesse, contendo três atributos: a localização, a data da coleta e o valor da variável coletada.

A inferência da complexidade média da operação de inserção foi feita pela medição do tempo de inserção de 100.000 (cem mil) registros de uma única variável, considerando sua posição geográfica (latitude e longitude), data de aquisição e valor, em ambos os bancos de dados. Os dados foram gerados aleatoriamente, com distribuição uniforme, usando a função nativa do Python. A inferência da função de complexidade das operações de consulta dos dados foi realizada sobre um banco de dados composto por 5 variáveis distintas e incrementando o número de registros no banco de dados, até 4.000 (quatro mil) registros, considerando, para cada uma das variáveis, sua localização (latitude e longitude), data de aquisição e o valor da variável.

As operações de consulta foram feitas de três formas distintas: (i) sobre todos os valores armazenados, (ii) somente valores próximos a um ponto e (iii) valor de todas as variáveis em um ponto específico. Em todos os casos foram feitas consultas envolvendo uma, duas, três quatro e cinco variáveis. O objetivo foi inferir o crescimento da função de consulta em função tanto da quantidade de valores no banco quanto do número de variáveis envolvidas. No segundo tipo de consulta buscaram-se os valores das variáveis de interesse em pontos dentro de um raio $r$ de distância a um ponto central. $\mathrm{O}$ valor de $r$ foi sendo incrementado até 10 quilômetros, em intervalos de 500 metros. Esse tipo de busca pode responder à questão "Quais as características desta região?". Outra consulta pertinente em agropecuária é quais são os locais com determinada característica ou as características de um determinado local. Para responder esse tipo de questionamento, foram realizadas consulta sobre o valor de uma variável em uma localização específica. Cada consulta foi repetida dez vezes para o cálculo da média do tempo de execução. 


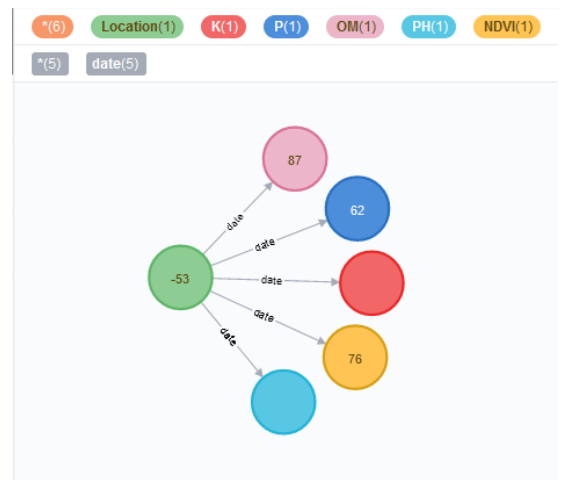

(a)

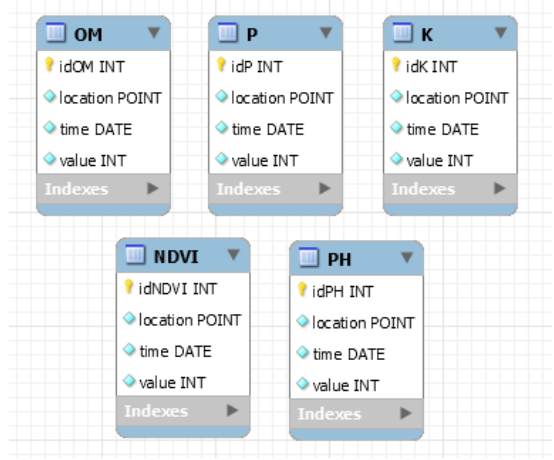

(b)

Figura 1. (a) Modelo de dados no Neo4j. (b) Modelo de dados no MySQL

O impacto no tempo de execução no número de elementos no banco de dados foi inferido com todas as consultas feiras com um incremento de 100 registros para cada variável a cada iteração do ciclo, até o máximo 4.000 elementos registrados para cada variável.

\section{RESULTADOS}

A inferência da complexidade de inserção nos bancos foi feita com cem mil inserções de uma única variável. Os resultados são apresentados na Figura 2. Para uma melhor visualização, foi calculada a média de mil inserções, totalizando cem pontos no gráfico. Os tempos de execução seguem o esperado de uma curva logarítmica, embora com as variações esperadas de um processo iterativo executado em uma máquina real. O tempo de inserção no banco Neo4J é maior do que no MySQL, o que também é esperado devido às eficientes estruturas de indexação (B-trees) usadas na implementação de bases relacionais.

Os tempos de execução das consultas em todos os elementos do banco para 1, 2, 3, 4 e 5 variáveis, com o tamanho do banco de dados sendo incrementado de $100 \mathrm{em} 100$ elementos aparecem na Figura 3. A Figura 3(a) apresenta os tempos de execução para a consulta no banco de dados MySQL e as curvas são consistentes com a operação de um produto cartesiano $n$-ário, que que o grau do polinômio que representa o tempo de execução cresce com o valor de $n$. Já para os dados armazenados no banco de dados de grafos, apresentado na Figura 3(b) o tempo de execução apresenta características lineares, com o coeficiente angular aumentando linearmente com a quantidade de variáveis. Esse resultado mostra que, para consultas sem restrições, o banco Neo4j mostra comportamento mais eficiente.

Os resultados das consultas a valores de variáveis restritos a uma distância fixa de um ponto central são apresentados na Figura 4. Novamente, o tempo médio das consultas foi calculado, para cada incremento de distância. A Figura 4(a) apresenta as curvas para o banco de dados MySQL, que seguem a mesma tendência das curvas de consulta a todos os elementos no banco de dados. A Figura 4(b) mostra as curvas para o banco Neo4J, que também refletem as consultas anteriores, com exceção da curva para uma variável, onde a média do tempo de execução aumenta também linearmente, com coeficiente angular maior que 1 . Novamente, o banco Neo4j apresenta-se assintoticamente mais eficiente que o banco MySQL. A influência da distância nas consultas é apresentada nas Figuras 5(a) e 5(b), com as curvas do tempo de execução das consultas em função do valor do raio $r$ e consequente aumento no número de pontos retornados nos bancos relacional e de grafos, respectivamente. Nessas consultas, somente o valor da distância $r$ variou: ambos os bancos tinham 4000 registros em cada variável. Como a consulta não exigia junções entre variáveis, as curvas do banco MySQL são todas lineares, com o coeficiente angular aumentando para cada variável inserida na consulta. As curvas para o banco Neo4j, apresentadas na Figura 5 (b), também seguem o mesmo padrão, mas com valores muito inferiores aos do banco MySQL (45 segundos contra 0,8 segundos, para consultas com 5 variáveis). 


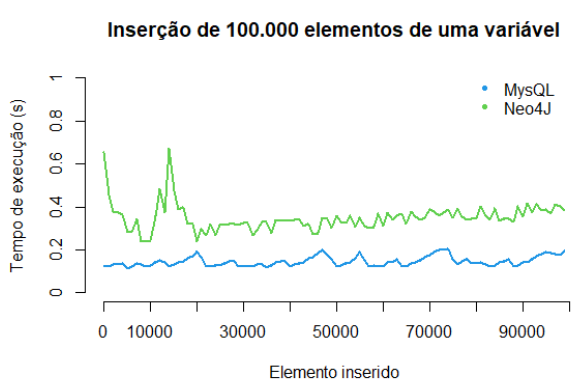

Figura 2. Tempos de execução da operação de inserção

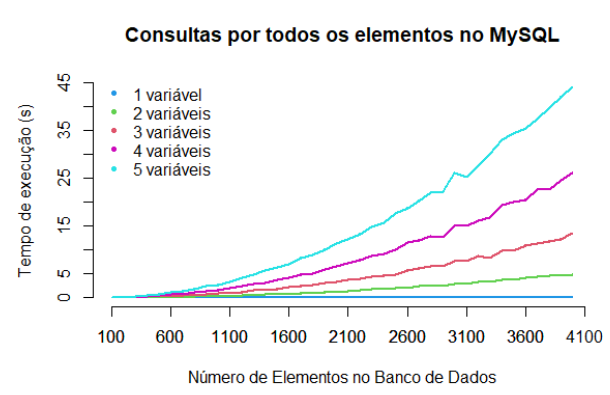

(a)

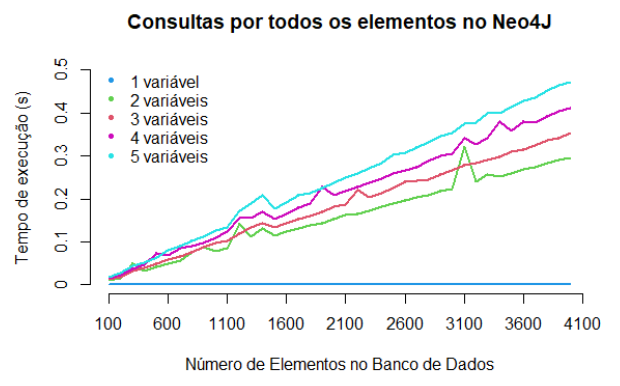

(b)

Figura 3. Tempos de execução da operação de consulta de todos os elementos no banco de dados MySQL (a) e Neo4J (b)

Consultas por distância no MySQL

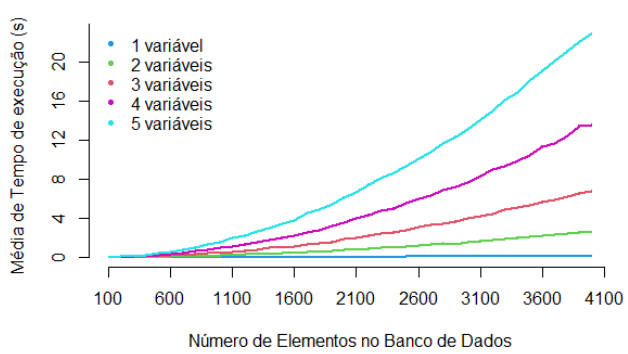

(a)

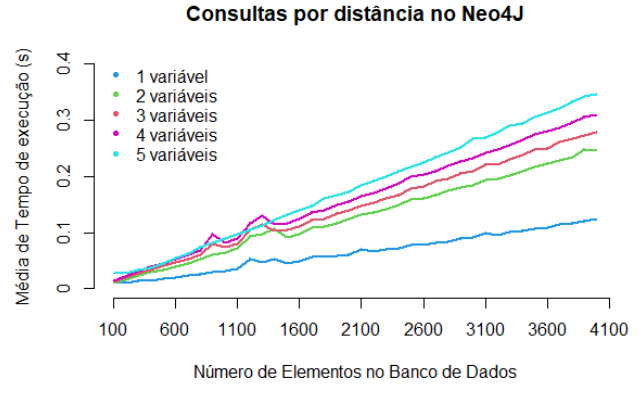

(b)

Figura 4. Tempos de execução de consulta a partir de um ponto central no banco MySQL (a) e Neo4J (b)

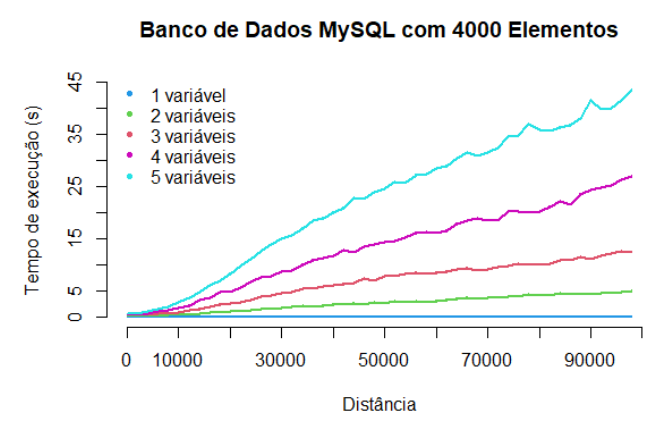

(a)

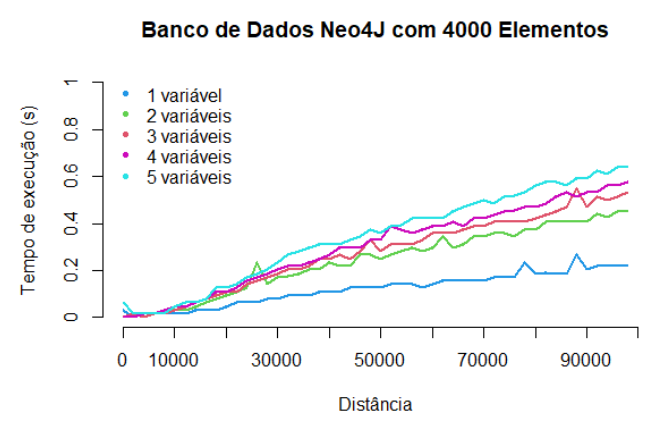

(b)

Figura 5. Tempos de execução das operações de consulta em função do raio $r$ no banco MySQL (a) e Neo4J (b) 


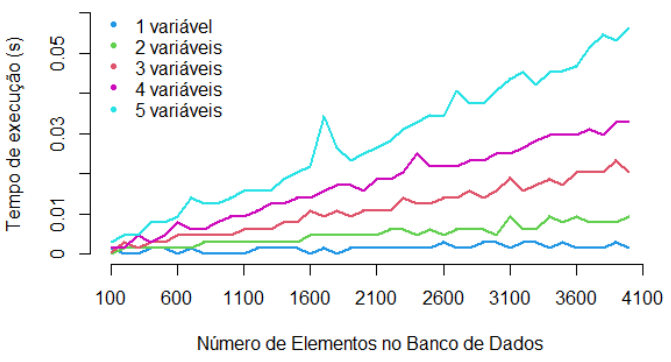

(a)

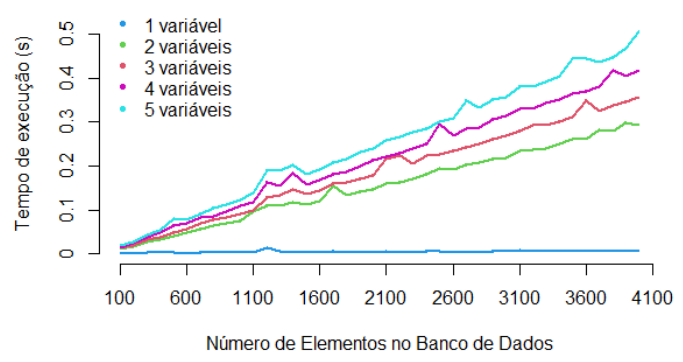

(b)

Figura 6. Tempos de execução das operações de consulta de valores de variáveis em um ponto específico em função do número de elementos no banco de dados MySQL (a) e Neo4J (b)

\section{CONCLUSÃO}

Com base nos resultados observados nos testes realizados, para o armazenamento de dados espaço-temporais de produção agrícola, a performance do banco de dados de grafos apresenta melhores resultados assintóticos, com exceção da operação de inserção. Como a inserção dos dados é realizada uma única vez, essa diferença torna-se pouco significativa ao longo do tempo. De forma geral, para dados que devem ser consultados tanto por sua chave quanto por valores de seus atributos, como é o caso em sistemas produtivos agropecuários, o desempenho de bancos de dados orientados a grafos, quando as operações são nativas, como é o caso do banco Neo4j, é significativamente melhor.

\section{REFERÊNCIAS}

Baker Effendi, Sedick, et al., 2020. "Suitability of Graph Database Technology for the Analysis of Spatio-Temporal Data". Future Internet, vol. 12, no 5, 2020.

Date, J. C. 2003. An Introduction to Database Systems. Pearson, Reino Unido.

Laksono, D., 2018. Testing spatial data deliverance in SQL and NOSQL database usingnodejs fullstack web app. In: Proceedings of the 4th International Conference onScience and Technology (ICST). pp. 1-5. Yogyakarta

Lian J. et. al, 2018. SQL or NoSQL? Which Is the Best Choice for Storing Big Spatio-Temporal Climate Data?Advances in Conceptual Modeling. ER 2018. Lecture Notes in Computer Science, vol. 11158. Springer.

MAPA, 2011. Agricultura de precisão. Ministério da Agricultura, Pecuária e Abastecimento, Brasília.

MySQL, 2020. Manual de referência do MySQL 4.1 https://downloads.mysql.com/docs/refman-4.1-pt.pdf

Python, 2020. The Python tutorial https://docs.python.org/3/tutorial/index.html

R Core Team, 2013. R: A Language and Environment for Statistical Computing. R Foundation for Statistical Computing, Vienna, Austria

Robinson, Ian et. al., 2015. Graph Database. O'Reilly, USA. 